\section{D) Check for updates}

Cite this: Nanoscale, 2020, 12, 14818

\title{
DNA density-dependent uptake of DNA origami- based two-or three-dimensional nanostructures by immune cells $\uparrow$
}

\author{
Tatsuoki Maezawa, (D) a Shozo Ohtsuki, (D) a Kumi Hidaka, ${ }^{b}$ Hiroshi Sugiyama, (D) b,c \\ Masayuki Endo, (ID b,c Yuki Takahashi, iD a Yoshinobu Takakura a and \\ Makiya Nishikawa (iD *a,d
}

\begin{abstract}
DNA nanostructures are expected to be applied for targeted drug delivery to immune cells. However, the structural properties of DNA nanostructures required for the delivery have not fully been elucidated. In this study, we focused on the DNA density that can be important for the their recognition and uptake by immune cells. To examine this, DNA nanostructures with almost identical molecular weights and structural flexibility, but with different shapes and DNA densities, were designed using DNA origami technology. We compared the following five types of DNA nanostructures, all of which consisted of ten DNA helices using an identical circular, single-stranded scaffold and staples. Rec180 had a rectangular-shaped, almost flat structure. Rec90, Rec50 and Rec0 were bent forms of Rec180 at the center by 90,50 or 0 degrees, respectively. Rec50/50 has two bends of 50 degrees each so that the both ends stick together to form a triangular prism shape. The fluctuation, or flexibility, of these DNA nanostructures under solution conditions was estimated using CanDo software. The DNA density estimated from the average distance between any two of the ten DNA helices in the DNA nanostructures was different among them; Rec50, Rec0 and Rec50/50 had a higher density than Rec180 and Rec90. Agarose gel electrophoresis and atomic force microscopy showed that all of the nanostructures were prepared with high yield. Flow cytometry analysis revealed that the uptake of DNA nanostructures by murine macrophage-like RAW264.7 cells was higher for those with higher DNA density than those with low density. There was a positive correlation between the density and cellular uptake. These results indicate that DNA nanostructures with high DNA density are suitable for delivery to immune cells.
\end{abstract}

Received 24th March 2020, Accepted 17th June 2020 DOI: $10.1039 / \mathrm{d} 0 \mathrm{nr} 02361 \mathrm{~b}$ rsc.li/nanoscale by utilizing hybridization between complementary sequences. At that time, however, it was difficult to control the size and form of DNA nanostructures. Therefore, the number of designable DNA nanostructures was limited. DNA origami technology was devised by Rothemund in 2006. In this technology, a long, single-strand DNA, a scaffold, and short "staple" DNAs complementary to parts of the scaffold are annealed into a targeted shape by self-assembly. ${ }^{3}$ This technology enables the preparation of various, perhaps arbitrary, DNA nanostructures, and many researchers accomplished the construction of various DNA nanostructures. ${ }^{4-6}$ In addition, versatile functions were added to DNA nanostructures by adding various molecules such as proteins and photo-responsive functional groups. ${ }^{7,8}$

DNA nanostructures are expected to be used in many fields, and are expected to play important roles in drug delivery, because DNA is biodegradable and biocompatible, which are suitable properties for delivery systems. Some researchers succeeded in drug delivery by using DNA nanostructures in vitro and in vivo. Schuller et al. demonstrated that DNA containing an unmethyl- 
ated cytosine-phosphate-guanine ( $\mathrm{CpG}$ ) motif connected to a tubular DNA nanostructure activated immune cells with higher efficiency than single-strand CpG DNA because of its accelerated cellular uptake. ${ }^{9}$ Jiang et al. loaded doxorubicin into tubular and triangular DNA nanostructures and demonstrated an enhanced delivery of the anticancer agent to human breast cancer-derived MCF7 cells. ${ }^{10}$ Douglas et al. developed a box-shaped DNA nanostructure sealed with aptamers against the platelet-derived growth factor (PDGF) and showed that it selectively delivered a conjugated antibody to human CD3 cells that expressed PDGF ${ }^{11}$

To develop DNA nanostructures that are suitable for drug delivery, it is important to elucidate the relationship between the structural properties of DNA nanostructures and their cellular uptake. However, despite many studies on the applications of DNA nanostructures to drug delivery, little information is available about the structural properties of DNA nanostructures suitable for cellular uptake.

We developed various DNA nanostructures including polypod-like structured DNA, or polypodna, ${ }^{12}$ polypodna-based DNA dendrimers, ${ }^{13}$ tetrahedral and tetragonal DNAs, ${ }^{14}$ and rectangular DNA nanostructures. ${ }^{15}$ By comparing their uptake by immune cells, it was found that DNA nanostructures with more complicated structures tended to be more effectively taken up by immune cells than simpler ones. In addition, this structuredependent uptake was almost specific to dendritic cells and macrophages, but was hardly applied to fibroblasts and endothelial cells. ${ }^{16}$ However, in these studies, multiple factors of DNA nanostructures, such as molecular weight and DNA density, were different among them. These factors could affect the cellular uptake of DNA nanostructures, which could make it difficult to elucidate the factors determining their cellular uptake.

In this study, we focused on the DNA density of DNA nanostructures and designed five types of DNA which have different DNA densities for one structure. We also paid attention to the molecular weight and structural flexibility, and designed DNA nanostructures with nearly identical molecular weights and structural flexibility to minimize the possible influence of these parameters. To this end, we designed five types of twoor three-dimensional DNA nanostructures using an identical circular, single-stranded scaffold and staples, each of which was bent at different angles to endow them with different DNA densities. By evaluating their structural properties and cellular uptake by murine macrophage-like RAW264.7 cells, we elucidated the relationship between the DNA density of DNA nanostructures and their uptake by the cells.

\section{Materials and methods}

\section{Chemicals}

RPMI1640 medium was purchased from Nissui Pharmaceutical Co., Ltd (Tokyo, Japan). Fetal bovine serum (FBS) and Optimodified Eagle's medium (Opti-MEM) were obtained from Thermo Fisher Scientific (Waltham, MA, USA). All of the other chemicals were of the highest grade available and used without further purification.

\section{Cell culture}

Murine macrophage-like RAW264.7 cells were obtained from ATCC (Manassas, VA, USA) and cultured in RPMI1640 medium supplemented with $10 \%$ heat-inactivated FBS, $0.2 \% \mathrm{NaHCO}_{3}$, 100 units per ml of penicillin, $100 \mu \mathrm{g} \mathrm{ml}^{-1}$ of streptomycin and $2 \mathrm{mM}$ L-glutamine at $37{ }^{\circ} \mathrm{C}$ in humidified air containing $5 \%$ $\mathrm{CO}_{2}$. The cells were then seeded on 96-well culture plates at a density of $5 \times 0^{4}$ cells per well and cultured for $24 \mathrm{~h}$ prior to use.

\section{Scaffold}

To construct DNA nanostructures with an optimal size for cellular uptake, a scaffold 1801 bases long developed in a previous study ${ }^{15}$ was used. The sequence of the scaffold is as follows:

5'-CTAGATACAAATGTGGTATGGAATTCAGTCAATATGTTCACCCCAAAAAAGCTGTTTGTTAACTTGCCAACCTCATTCTAAAATGTATATAGAAGCCCAAAAGACAATAACAAAAATATTCTTGTAGAACAAAATGGGAAAGAATGTTCCACTAAATATCAAGATTTAGAGCAAAGCATGAGATGTGTGGGGATAGACAGTGAGGCTGATAAAATAGAGTAGAGCTCAGAAACAGACCCATTGATATATGTAAGTGACCTATGAAAAAAATATGGCATTTTACAATGGGAAAATGATGATCTTTTTCTTTTTTAGAAAAACAGGGAAATATATTTATATGTAAAAAATAAAAGGGAACCCATATGTCATACCATACACACAAAAAAATTCCAGTGAATTATAAGTCTAAATGGAGAAGGCAAAACTTTAAATCTTTTAGAAAATAATATAGAAGCATGCCATCAAGACTTCAGTGTAGAGAAAAATTTCTTATGACTCAAAGTCCTAACCACAAAGAAAAGATTGTTAATTAGATTGCATGAATATTAAGACTTATTTTTAAAATTAAAAAACCATTAAGAAAAGTCAGGCCATAGAATGACAGAAAATATTTGCAACACCCCAGTAAAGAGAATTGTAATATGCAGATTATAAAAAGAAGTCTTACAAATCAGTAAAAAATAAAACTAGACAAAAATTTGAACAGATGAAAGAGAAACTCTAAATAATCATTACACATGAGAAACTCAATCTCAGAAATCAGAGAACTATCATTGCATATACACTAAATTAGAGAAATATTAAAAGGCTAAGTAACATCTGTGGCTTAATTAAAACAGGTAGTTGACAATTAAACATTGGCATAGTATATCTGCATAGTATAATACAACTCACTATAGGAGGGCCATCATGGCCAAGTTGACCAGTGCTGTCCCAGTGCTCACAGCCAGGGATGTGGCTGGAGCTGTTGAGTTCTGGACTGACAGGTTGGGGTTCTCCAGAGATTTTGTGGAGGATGACTTTGCAGGTGTGGTCAGAGATGATGTCACCCTGTTCATCTCAGCAGTCCAGGACCAGGTGGTGCCTGACAACACCCTGGCTTGGGTGTGGGTGAGAGGACTGGATGAGCTGTATGCTGAGTGGAGTGAGGTGGTCTCCACCAACTTCAGGGATGCCAGTGGCCCTGCCATGACAGAGATTGGAGAGCAGCCCTGGGGGAGAGAGTTTGCCCTGAGAGACCCAGCAGGCAACTGTGTGCACTTTGTGGCAGAGGAGCAGGACTGAGGATAACCTAGGAAACCTTAAAACCTTTAAAAGCCTTATATATTCTTTTTTTTCTTATAAAACTTAAAACCTTAGAGGCTATTTAAGTTGCTGATTTATATTAATTTTATTGTTCAAACATGAGAGCTTAGTACATGAAACATGAGAGCTTAGTACATTAGCCATGAGAGCTTAGTACATTAGCCATGAGGGTTTAGTTCATTAAACATGAGAGCTTAGTACATTAAACATGAGAGCTTAGTACATACTATCAACAGGTTGAACTGCTGATCTTAATTAAAATTATCTCTAAGGCATGTGAACTGGCTGTCTTGGTTTTCATCTGTACTTCATCTGCTACCTCTGTGACCTGAAACATATTTATAATTCCATTAAGCTGTGCATATGATAGATTTATCATATGTATTTTCCTTAAAGGATTTTTGTAAGAACTAATTGAATTGATACCTGTAAAGTCTTTATCACACTACCCAATAAATAATAAATCTCTTTGTTCAGCTCTCTGTTTCGCTGAGGT-3'. 


\section{Staples}

Staples, short single-strand DNA 16-50 bases long, were purchased from Eurofin Genomics Japan K.K. (Tokyo, Japan). Ten staples (two staples for each DNA nanostructure) labeled with Alexa Fluor488 at the 5'-end were purchased from Japan BioService Co.,Ltd (Saitama, Japan). Sequences of staples used in DNA nanostructures are listed in ESI, Table S1.†

\section{Design of DNA nanostructures}

DNA nanostructures were designed using caDNAno, computeraided design software of two- and three-dimensional DNA nanostructures as previously reported. ${ }^{2}$

\section{Fluctuation or flexibility of DNA nanostructures}

The JavaScript Object Notation files of the five DNA nanostructures were prepared using caDNAno and analyzed in CanDo, a DNA nanostructure's model generating system. ${ }^{3}$ In CanDo analysis, the DNA nanostructure is divided into hundreds of parts, and all conformations speculated to exist in solution at room temperature are calculated. Based on this calculation, the root mean square fluctuation (RMSF) of each part is determined as a parameter of fluctuation or flexibility.

\section{DNA density of DNA nanostructures}

The average distance between two of the DNA helices composing the DNA nanostructures was used as an index of DNA density of DNA nanostructures.

\section{Preparation of DNA nanostructures}

Each structure was prepared by mixing equimolar amounts of the scaffold and 50 staples. The scaffold and the staples dissolved in a $\mathrm{TE}-\mathrm{Mg}^{2+}$ buffer $(20 \mathrm{mM}$ Tris-HCl, $1 \mathrm{mM}$ ethylenediaminetetraacetic acid (EDTA), and $10 \mathrm{mM}$ magnesium chloride, $\mathrm{pH}$ 7.3) were mixed at a final concentration of $60 \mathrm{nM}$ for each DNA. When preparing fluorescently labeled DNA nanostructures, staples labeled with Alexa Fluor488 were mixed instead of unlabeled staples. The mixtures were then heated to $85{ }^{\circ} \mathrm{C}$ and gradually cooled down to $4{ }^{\circ} \mathrm{C}$ using a thermal cycler (PC-818S Program Temp Control System; ASTEC Co., Ltd, Fukuoka, Japan). The products were analyzed by agarose gel electrophoresis in $0.5 \times$ TBE buffer containing $10 \mathrm{mM}$ potassium (45 mM Tris borate, $1 \mathrm{mM}$ EDTA, supplemented with $10 \mathrm{mM} \mathrm{MgCl}_{2}$ ) at $4{ }^{\circ} \mathrm{C}$. The gel was stained with SYBR Gold (Thermo Fisher Scientific) and observed using a LAS3000 (Fujifilm, Tokyo, Japan).

\section{AFM imaging of DNA nanostructures}

Atomic force microscopy (AFM) images of each structure were obtained using an AFM system (Nano Live Vision, RIBM, Tsukuba, Japan) using a silicon nitride cantilever (BL-AC10EGS; Olympus, Tokyo, Japan). In brief, a DNA sample was adsorbed on a freshly cleaved mica plate pretreated with $0.1 \%$ aqueous 3 -aminopropyltriethoxysilane for $5 \mathrm{~min}$ at room temperature and then washed three times with a buffer solu- tion containing $20 \mathrm{mM}$ Tris and $10 \mathrm{mM} \mathrm{MgCl}$. Scanning was performed in the same buffer solution.

\section{Flow cytometry}

Alexa Fluor488-labeled single-stranded DNA (ssDNA) and five DNA nanostructures were diluted in $50 \mu \mathrm{l}$ Opti-MEM to $3 \mathrm{nM}$. They were added to RAW264.7 cells on a 96-well plate at a density of $5 \times 10^{4}$ cells per well and incubated for $4 \mathrm{~h}$ at $37^{\circ} \mathrm{C}$ or $4{ }^{\circ} \mathrm{C}$. RAW264.7 cells were washed three times with PBS and harvested. Thereafter, the fluorescence intensity of cells was determined by flow cytometry (Gallios Flow Cytometer, BD Biosciences, NJ, USA) using Kaluza software (version 1.0, BD Biosciences), and the mean fluorescence intensity (MFI) was calculated.

\section{Statistical analysis}

Differences were statistically evaluated by one-way analysis of variance (ANOVA) followed by the Tukey-Kramer test for multiple comparisons. A $p$ value less than 0.05 was considered statistically significant.

\section{Results and discussion}

\section{Design of DNA nanostructures}

We firstly designed Rec180, an almost flat, rectangular-shaped DNA nanostructure, as reported previously. ${ }^{15}$ Assuming the helical diameter of DNA, the interhelical gap, and the distance between bases in double-strand DNA were 2, 0.5 and $0.34 \mathrm{~nm}$, respectively, Rec180 has $24.5 \mathrm{~nm}$ width (10 helix), $54.1 \mathrm{~nm}$ height (160 base), and $2 \mathrm{~nm}$ depth (1 helix). There are singlestranded loops on both sides of Rec180 so that the interaction between the DNA nanostructures through $\pi-\pi$ stacking hardly occurs. Then, based on the design of Rec180, four DNA nanostructures were designed. Rec180 was bent at the center by 90 , 50 or 0 degrees to obtain Rec 90 , Rec50 and Rec0, respectively, or bent twice by 50 degrees each to obtain Rec50/50. Schematic illustrations of these DNA nanostructures are shown in Fig. 1. They are constructed using the same 1801-base long scaffold and 50 staples, and the total base number per nanostructure was identical to that of Rec180. Therefore, the molecular weights of the five DNA nanostructures designed were almost identical.

\section{Structural flexibility of DNA nanostructures}

The proportion of the single- and double-stranded parts is important for root mean square fluctuation (RMSF) or structural flexibility of DNA nanostructures. Actually, reducing the number of staples and increasing single-stranded parts result in larger RMSF values. Fig. 2 shows the results of CanDo analysis of each DNA nanostructure. Both sides of the DNA nanostructures were single stranded. Therefore, the RMSF values of the sides were relatively high, indicating that these parts are flexible. On the other hand, the central part of the DNA nanostructures, where there are many crossovers between double helices, had low RMSF values, indicating that this part is a 

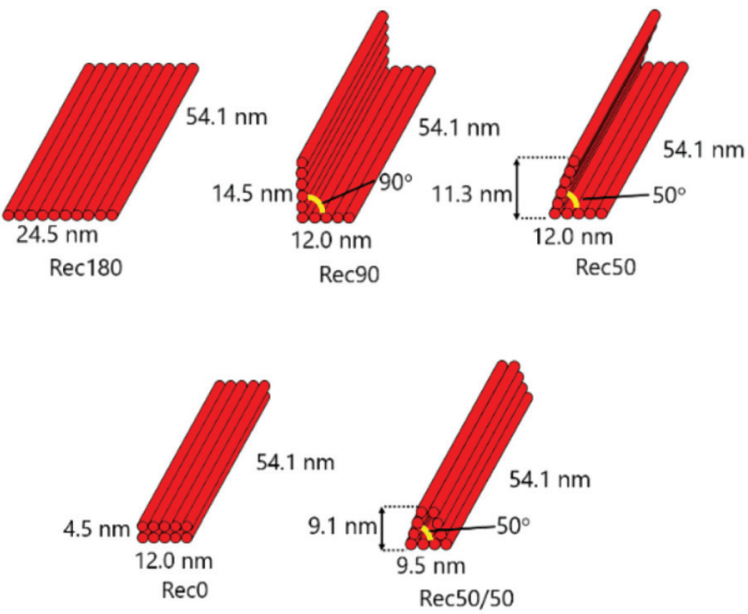

Fig. 1 Designs of Rec180, Rec90, Rec50, Rec0 and Rec50/50. Rec180 is a rectangular-shaped DNA nanostructure. Rec180 was bent at the center by 90,50 or 0 degrees to obtain Rec 90 , Rec50 and Rec0, respectively, or bent twice by 50 degrees each for Rec50/50.

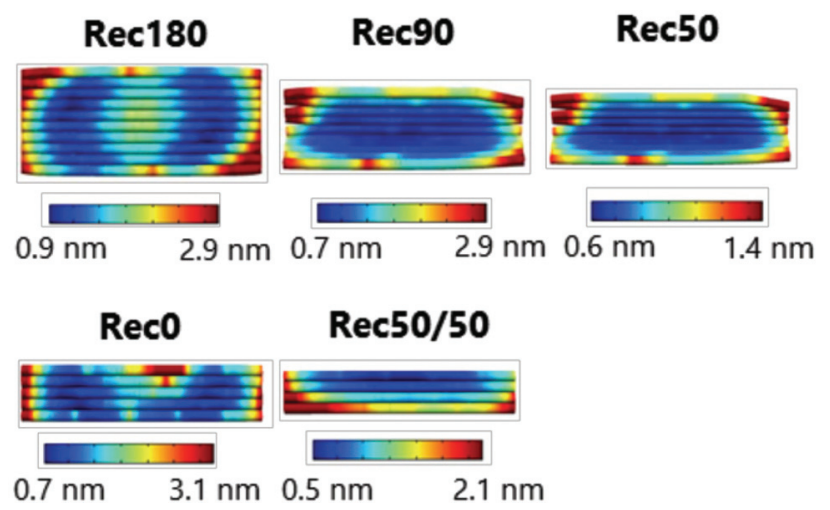

Fig. 2 Results of CanDo analysis of the DNA nanostructures. Each scale bar means the root mean square fluctuation (RMSF) of each part. In the areas colored by red, RMSF is relatively high, indicating that they are flexible. In the areas colored by blue, RMSF is relatively low, indicating that this part is a rigid structure.

rigid structure. These characteristics were common to all the DNA nanostructures prepared. This could be due to the fact that they were prepared using an identical scaffold and 50 staples. A slightly higher RMSF value of the central part of Rec180 could be explained by the fact that this part has few crossovers (ESI, $\uparrow$ Fig. 1). Our previous study showed that DNA nanostructures prepared with different numbers of staples possessed different structural flexibilities depending on the number and position of the staples. ${ }^{15}$ Therefore, these results suggest that the flexibility is comparable among the DNA nanostructures designed.

\section{DNA density of DNA nanostructures}

Fig. 3a shows the calculation method for the average distance between DNA helices and the average distance between the DNA helices of each nanostructure. Each DNA nanostructure a

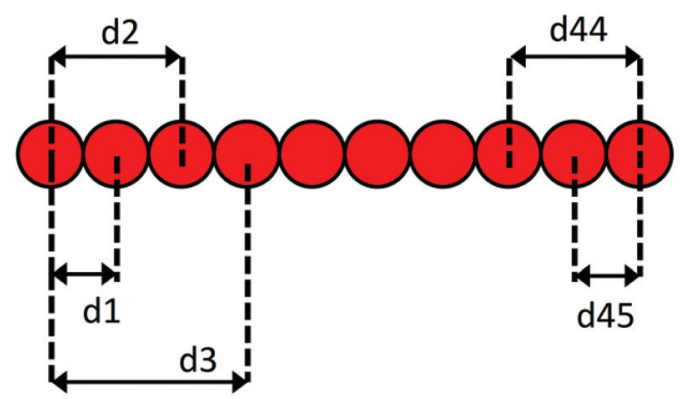

Average distance between DNA helices

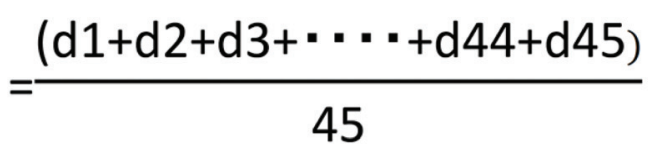

b

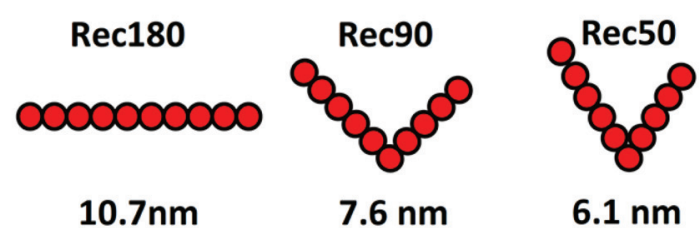

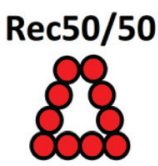

$5.1 \mathrm{~nm}$

Fig. 3 (a) Calculation method of the average distance between DNA helices. (b) Arrangement of helices and the average distance between DNA helices of each DNA nanostructure.

consists of 10 helices, so that there are 45 combinations of the two helices in total. The average distances of the five DNA nanostructures calculated were $10.7,7.1,6.2,5.0$, and $5.1 \mathrm{~nm}$ for Rec180, Rec90, Rec50, Rec0, and Rec50/50, respectively (Fig. 3b).

\section{Agarose gel electrophoresis of DNA nanostructures}

Fig. 4 shows the agarose gel electrophoresis analysis of the five DNA nanostructures. The bands of the DNA nanostructures were slightly higher than those of the scaffold, and the band of the staples was hardly detected in all the DNA nanostructures. These results indicate that the scaffold and the staples hybridize to form each DNA nanostructure.

\section{AFM observation of DNA nanostructures}

Fig. 5 shows the AFM images of the five DNA nanostructures. These images were obtained after the adsorption of the DNA nanostructure onto mica plates. Therefore, both the two- and three-dimensional DNA nanostructures should be observed as 


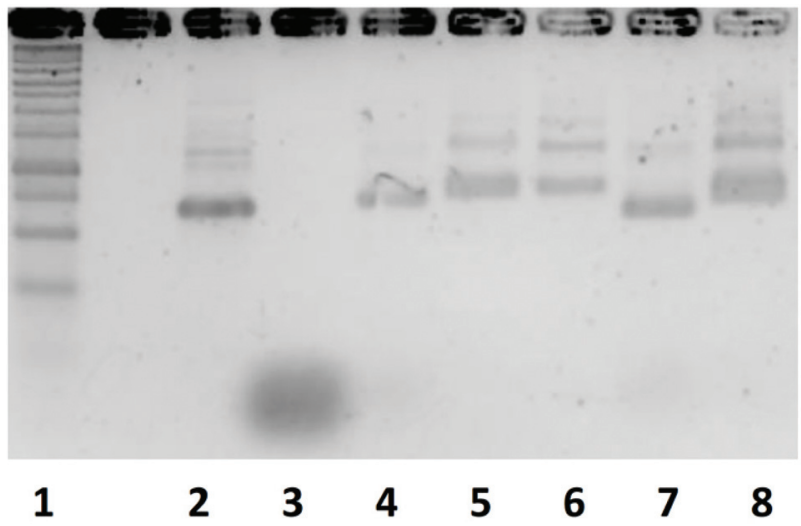

Fig. 4 Agarose gel electrophoresis analysis of an assembled DNA nanostructure. Lane1, 1kbp DNA ladder; Lane2, scaffold; Lane3, staple; Lane4 Rec180; Lane5, Rec90; Lane6, Rec50; Lane7, Rec0; Lane8, Rec50/50.

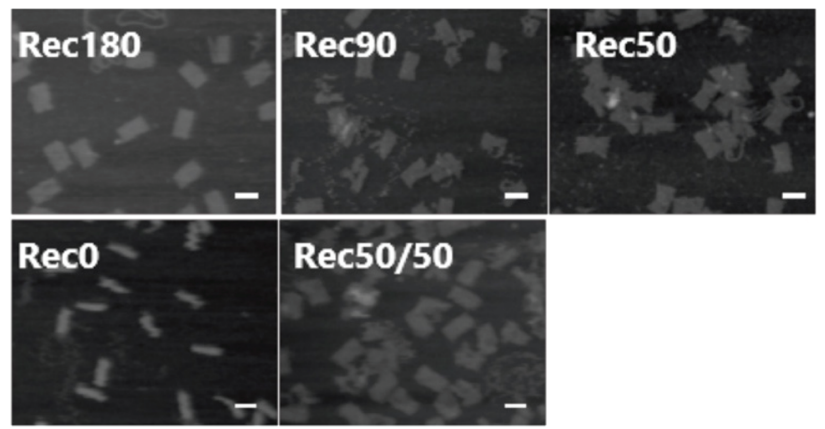

Fig. 5 AFM images of Rec180, Rec90, Rec50, Rec0, and Rec50/50. Scale bar $=50 \mathrm{~nm}$.

two-dimensional shapes. Rec0 was observed as a rectangle with about $12.5 \mathrm{~nm}$ width and about $50 \mathrm{~nm}$ height. Other DNA nanostructures were also observed as rectangular shapes with about $25 \mathrm{~nm}$ width and about $50 \mathrm{~nm}$ height. Some collapsed or unfolded structures were observed in the images, but their percentage was low in all the DNA nanostructures. These results of the electrophoresis and AFM imaging indicate that all the DNA nanostructures are constructed as designed with high yield.

\section{Cellular uptake of DNA nanostructures}

Fig. 6 shows the uptake of the DNA nanostructures by RAW264.7 cells. When RAW264.7 cells were incubated at $37^{\circ} \mathrm{C}$, all the DNA nanostructures were taken up by the cells much more efficiently than single-stranded DNA. The uptake of Rec50/50 was the highest. The DNA nanostructures with high DNA density tended to be taken up more efficiently than those with low density. When RAW264.7 cells were incubated at $4{ }^{\circ} \mathrm{C}$, cellular uptake of all DNA nanostructures was comparable (data not shown). Fig. 7 shows the relationship between the average distance between DNA helices and the uptake by RAW264.7 cells of the DNA nanostructures. There was a negative correlation between these two, suggesting that the DNA

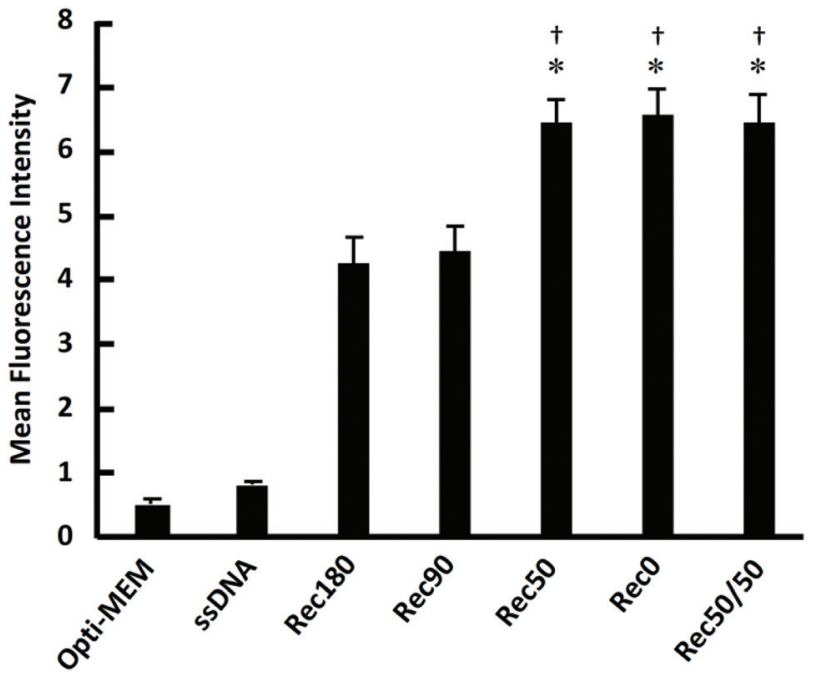

Fig. 6 Cellular uptake of DNA nanostructures. Each Alexa Fluor488labeled DNA sample was added to cells at $3 \mathrm{nM}$. Results are expressed as the ratio of MFI of four wells. ${ }^{*} p<0.05$ vs. Rec180 $\dagger p<0.05$ vs. Rec 90 .

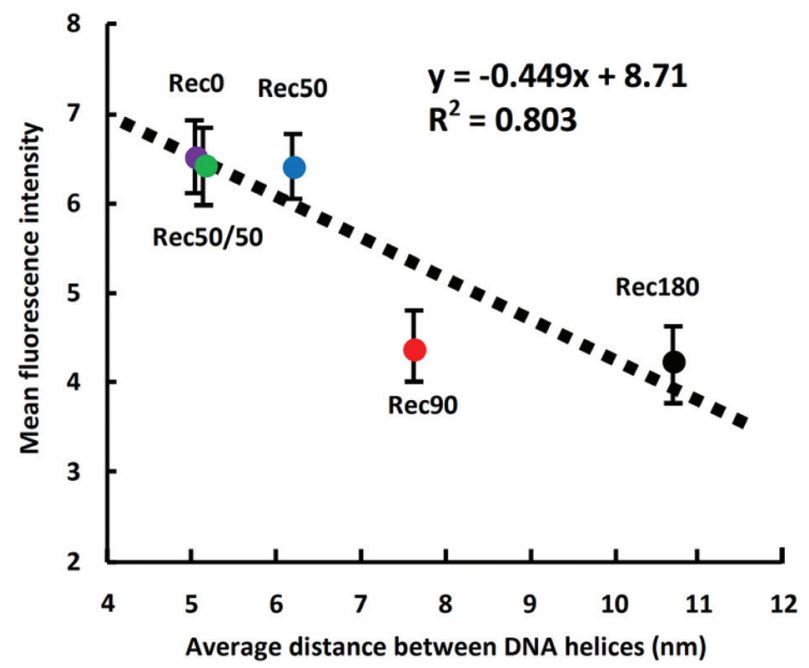

Fig. 7 Correlation between the average distance between DNA helices and uptake by RAW264.7 cells. The smaller the average distance between DNA helices of a DNA nanostructure, the higher the DNA density of the DNA nanostructure and the more efficient uptake by RAW264.7 cells.

density is a key parameter determining the cellular uptake when the molecular weight and structural flexibility were comparable to one another.

Most studies on the DNA origami technology use M13mp18 (p7249) single-stranded DNA of 7249 bases as a scaffold. It is because the source of a long single-stranded DNA other than this is limited. However, the size of M13mp18 is relatively large, and M13mp18-based DNA nanostructures are generally larger than $100 \mathrm{~nm}$ in length. Taking into consideration that the upper size limit of endocytotic uptake is around $100 \mathrm{~nm},{ }^{17}$ shorter single-stranded DNAs are suitable as a scaffold to 
evaluate the relationship between the structural properties and cellular uptake of DNA nanostructures. Therefore, we used a scaffold of 1801 bases which was previously constructed, ${ }^{15}$ and prepared five DNA nanostructures with a suitable size for cellular uptake. The sizes of these DNA nanostructures are comparable to the size of nanosized drug delivery systems, such as polymeric micelles and liposomes.

TLR9 receptor-positive immune cells would release proinflammatory cytokines upon stimulation with CpG DNA, and these cytokines could affect the cellular uptake of compounds, including DNA nanostructures. Therefore, the DNA nanostructures used should contain a few or no CpG motifs. pCpGmcs, a CpG-free plasmid DNA, was used as the template for the preparation of the scaffold to accomplish this.

In this study, DNA nanostructures with high DNA density were more efficiently taken up by RAW264.7 cells than those with low DNA density. In contrast, little difference was observed in the MFI values at $4{ }^{\circ} \mathrm{C}$ among the DNA nanostructures. These findings suggest that the DNA density of DNA nanostructures affects the cellular uptake, especially the rate of endocytosis. The cellular uptakes of Rec50, Rec0, and Rec50/50 were comparable to one another, although the average distance between DNA helices was slightly different. These results may suggest that there could be an upper limit in the cellular uptake efficacy when the DNA density exceeds a certain value. In general, larger DNA nanostructures are more efficiently taken up by smaller ones. The results of the present study, however, show that the DNA density is another important parameter than the size, because the smaller ones, i.e., Rec50/50 and Rec0, were most efficiently taken up by the cells.

There are several reports about the receptors involved in the cellular uptake of DNA and DNA nanostructures. In these studies, scavenger receptors, ${ }^{18}$ membrane-anchored protein $1,{ }^{19}$ receptors for advanced glycation end product, ${ }^{20}$ membrane-associated nucleic acid-binding protein (MNAB), ${ }^{21}$ mannose receptor $\mathrm{C}$ type $1^{22}$ and lymphocyte antigen 75 $\left(\right.$ DEC-205) ${ }^{23}$ are reported to be important for DNA uptake. We demonstrated that the transfection of human macrophage scavenger receptor 1 to HEK-Blue HTLR9 cells greatly increased the uptake of both DNA and DNA nanostructures. ${ }^{24}$ It was also demonstrated that the uptake of complicated DNA nanostructures was more efficient than that of simple ones. Therefore, it is expected that, by using the evaluation method utilized in these studies, the relationship between the interaction of DNA nanostructures with receptors and their structural properties such as DNA density can be elucidated.

\section{Conclusion}

In conclusion, the present study suggests that the cellular uptake of DNA nanostructures with high DNA density tends to be more efficient than that of DNA nanostructures with low DNA density. This result will provide useful information for the development of DNA nanostructure-based delivery systems for nucleic acid medicines to immune cells.

\section{Conflicts of interest}

There are no conflicts to declare.

\section{Acknowledgements}

This work was supported in part by JST CREST Grant Number JPMJCR1521, Japan, and Grants-in-Aid for Scientific Research (B) (23390010 and 26293008) from the Japan Society for the Promotion of Science.

\section{References}

1 N. C. Seeman, J. Theor. Biol., 1982, 99, 237-247.

2 E. Winfree, F. Liu, L. A. Wenzler and N. C. Seeman, Nature, 1998, 394, 539-544.

3 P. W. Rothemund, Nature, 2006, 440, 297-302.

4 R. Veneziano, S. Ratanalert, K. Zhang, F. Zhang, H. Yan, W. Chiu and M. Bathe, Science, 2016, 352, 1534.

5 D. Han, S. Pal, J. Nangreave, Z. Deng, Y. Liu and H. Yan, Science, 2011, 332, 342-346.

6 H. Dietz, S. M. Douglas and W. M. Shih, Science, 2009, 325, 725-730.

7 T. Masubuchi, M. Endo, R. Iizuka, A. Iguchi, D. H. Yoon, T. Sekiguchi, H. Qi, R. Iinuma, Y. Miyazono, S. Shoji, T. Funatsu, H. Sugiyama, Y. Harada, T. Ueda and H. Tadakuma, Nat. Nanotechnol., 2018, 13, 933-940.

8 E. M. Willner, Y. Kamada, Y. Suzuki, T. Emura, K. Hidaka, H. Dietz, H. Sugiyama and M. Endo, Angew. Chem., Int. Ed., 2017, 56, 15324-15328.

9 V. J. Schuller, S. Heidegger, N. Sandholzer, P. C. Nickels, N. A. Suhartha, S. Endres, C. Bourquin and T. Liedl, ACS Nano, 2011, 5, 9696-9702.

10 Q. Jiang, C. Song, J. Nangreave, X. Liu, L. Lin, D. Qiu, Z. G. Wang, G. Zou, X. Liang, H. Yan and B. Ding, J. Am. Chem. Soc., 2012, 134, 13396-13403.

11 S. M. Douglas, I. Bachelet and G. M. Church, Science, 2012, 335, 831-834.

12 K. Mohri, M. Nishikawa, N. Takahashi, T. Shiomi, N. Matsuoka, K. Ogawa, M. Endo, K. Hidaka, H. Sugiyama, Y. Takahashi and Y. Takakura, ACS Nano, 2012, 6, 59315940.

13 K. Mohri, E. Kusuki, S. Ohtsuki, N. Takahashi, M. Endo, K. Hidaka, H. Sugiyama, Y. Takahashi, Y. Takakura and M. Nishikawa, Biomacromolecules, 2015, 16, 1095-1101.

14 S. Ohtsuki, N. Matsuzaki, K. Mohri, M. Endo, T. Emura, K. Hidaka, H. Sugiyama, Y. Takahashi, K. Ishiyama, N. Kadowaki, Y. Takakura and M. Nishikawa, Nucleic Acid Ther., 2015, 25, 245-253.

15 S. Ohtsuki, Y. Shiba, T. Maezawa, K. Hidaka, H. Sugiyama, M. Endo, Y. Takahashi, Y. Takakura and M. Nishikawa, Nanoscale, 2019, 11, 23416-23422. 
16 K. Mohri, K. Nagata, S. Ohtsuki, S. Toyama, M. Nonomura, Y. Takahashi, Y. Takakura, M. Nishikawa and S. Sakuma, Pharm. Res., 2017, 34, 2362-2370.

17 R. A. Petros and J. M. DeSimone, Nat. Rev. Drug Discovery, 2010, 9, 615-627.

18 Y. Kimura, K. Sonehara, E. Kuramoto, T. Makino, S. Yamamoto, T. Yamamoto, T. Kataoka and T. Tokunaga, J. Biochem., 1994, 116, 991-994.

19 L. Benimetskaya, J. D. Loike, Z. Khaled, G. Loike, S. C. Silverstein, L. Cao, J. E. L. Khoury, T. Q. Cai and C. A. Stein, Nat. Med., 1997, 3, 414-420.

20 C. M. Sirois, T. Jin, A. L. Miller, D. Bertheloot, H. Nakamura, G. L. Horvath, A. Mian, J. Jiang, J. Schrum, L. Bossaller, K. Pelka, N. Garbi, Y. Brewah, J. Tian, C. Chang, P. S. Chowdhury, G. P. Sims, R. Kolbeck, A. J. Coyle, A. A. Humbles, T. S. Xiao and E. Latz, J. Exp. Med., 2013, 210, 2447-2463.
21 D. C. Siess, C. T. Vedder, L. S. Merkens, T. Tanaka, A. C. Freed, S. L. McCoy, M. C. Heinrich, M. E. Deffebach, R. M. Bennett and S. H. Hefeneider, J. Biol. Chem., 2000, 275, 33655-33662.

22 A. P. Moseman, E. A. Moseman, S. Schworer, I. Smirnova, T. Volkova, U. von Andrian and A. Poltorak, J. Immunol., 2013, 191, 5615-5624.

23 M. H. Lahoud, F. Ahmet, J. G. Zhang, S. Meuter, A. N. Policheni, S. Kitsoulis, C. N. Lee, M. O’Keeffe, L. C. Sullivan, A. G. Brooks, R. Berry, J. Rossjohn, J. D. Mintern, J. Vega-Ramos, J. A. Villadangos, N. A. Nicola, M. C. Nussenzweig, K. J. Stacey, K. Shortman, W. R. Heath and I. Caminschi, Proc. Natl. Acad. Sci. U. S. A., 2012, 109, 16270-16275.

24 S. Ohtsuki, Y. Takahashi, T. Inoue, Y. Takakura and M. Nishikawa, Sci. Rep., 2017, 7, 13661. 